\title{
Erratum to: Poor tuberculosis treatment outcomes in Southern Mozambique (2011-2012)
}

\author{
Alberto L. García-Basteiro 1,2,3*, Durval Respeito ${ }^{1}$, Orvalho J. Augusto ${ }^{1}$, Elisa López-Varela ${ }^{1,2}$, Charfudin Sacoor ${ }^{1}$, \\ Victor G. Sequera², Aina Casellas², Quique Bassat ${ }^{1,2}$, Ivan Manhiça ${ }^{4}$, Eusebio Macete ${ }^{2}$, Frank Cobelens ${ }^{3}$ \\ and Pedro L. Alonso ${ }^{1,2}$
}

\section{Erratum}

Following publication of the original article [1], the authors became aware that a sentence in the abstract was not corrected from a previous version of the manuscript.

Instead of: "HIV infection (OR 2.73; 95 \% CI: 1.704.38), being female (OR: 1.39; $95 \%$ CI 1.31-1.91) and lack of laboratory confirmation (OR: 1.51; 95 \% CI 1.10$2.08)$ were associated with dying during the course of TB treatment ( $p$ value $<0.05)$ ".

It should read: "HIV infection (OR 2.73; 95 \% CI: 1.704.38), being male (OR: 1.39; $95 \%$ CI 1.01-1.91) and lack of laboratory confirmation (OR: 1.54; $95 \%$ CI 1.12-2.13) were associated with dying during the course of $\mathrm{TB}$ treatment ( $p$ value $<0.05)$ ".

Please note that none of the conclusions of this manuscript are altered as a result of this modification.

\section{Author details}

${ }^{1}$ Centro de Investigação em Saude de Manhiça (CISM), Maputo,

Mozambique. ${ }^{2}$ ISGlobal, Barcelona Ctr. Int. Health Res. (CRESIB), Hospital Clínic - Universitat de Barcelona, Barcelona, Spain. ${ }^{3}$ Amsterdam Institute for Global Health and Development, Academic Medical Centre, Amsterdam, The Netherlands. ${ }^{4}$ Ministry of Health, National Tuberculosis Program, Maputo, Mozambique.

Received: 23 August 2016 Accepted: 20 October 2016 Published online: 25 October 2016

\section{Reference}

1. García-Basteiro AL, et al. Poor tuberculosis treatment outcomes in Southern Mozambique (2011-2012). BMC Infect Dis. 2016;16:214. doi:10.1186/s12879016-1534-y.

\footnotetext{
* Correspondence: alberto.garcia-basteiro@manhica.net

${ }^{1}$ Centro de Investigação em Saude de Manhiça (CISM), Maputo,

Mozambique

${ }^{2}$ ISGlobal, Barcelona Ctr. Int. Health Res. (CRESIB), Hospital Clínic - Universitat

de Barcelona, Barcelona, Spain
} 